\title{
Introduction: surgical management of skull base meningiomas
}

\author{
Gabriel Zada, MD, MS, ${ }^{1}$ Mustafa K Başkaya, MD, ${ }^{2}$ and Mitesh V. Shah, MD $^{3}$ \\ ${ }^{1}$ Department of Neurosurgery, Keck School of Medicine, University of Southern California, Los Angeles, California; ${ }^{2}$ Department \\ of Neurosurgery, University of Wisconsin School of Medicine and Public Health, Madison, Wisconsin; and ${ }^{3}$ Department of \\ Neurosurgery, Goodman Campbell Brain and Spine Center, Indiana University School of Medicine, Indianapolis, Indiana
}

Meningiomas represent the most common primary intracranial neoplasm treated by neurosurgeons. Although multimodal treatment of meningiomas includes surgery, radiation-based treatments, and occasionally medical therapy, surgery remains the mainstay of treatment for most symptomatic meningiomas. Because of the intricate relationship of the dura mater and arachnoid mater with the central nervous system and cranial nerves, meningiomas can arise anywhere along the skull base or convexities, and occasionally even within the ventricular system, thereby mandating a catalog of surgical approaches that neurosurgeons may employ to individualize treatment for patients. Skull base meningiomas represent some of the most challenging pathology encountered by neurosurgeons, on account of their depth, invasion, vascularity, texture/consistency, and their relationship to bony anatomy, cranial nerves, and blood vessels. Resection of complex skull base meningiomas often mandates adequate bony removal to achieve sufficient exposure of the tumor and surrounding region, in order to minimize brain retraction and optimally identify, protect, control, and manipulate sensitive neurovascular structures. A variety of traditional skull base approaches has evolved to address complex skull base tumors, of which meningiomas are considered the paragon in terms of both complexity and frequency.

In this supplemental video issue of Neurosurgical Focus, contributing authors from around the world provide instructiona narratives demonstrating resection of a variety of skull base meningiomas arising from traditionally challenging origins, including the clinoid processes, tuberculum sellae, dorsum sellae, petroclival region, falco-tentorial region, cerebellopontine angle, and foramen magnum. In addition, two cases of extended endoscopic endonasal approaches for tuberculum sellae and dorsum sellae meningiomas are presented, representing the latest evolution in accessing the skull base for selected tumors. Along with key pearls for safe tumor resection, an equally important component of open and endoscopic skull base operations for meningiomas addressed by the contributing authors is the reconstruction aspect, which must be performed meticulously to prevent delayed cerebrospinal fluid leakage and/or infections. This curated assortment of instructional videos represents the authors' optimal treatment paradigms pertaining to the selection of approach, setup, exposure, and principles to guide tumor resection for a wide spectrum of complex meningiomas.

KEYWORDS meningioma; neurosurgery; approaches; skull base; tumor; endoscopy

INCLUDE WHEN CITING Published online October 1, 2017; DOI: http://thejns.org/doi/abs/10.3171/2017.10.FocusVid.Intro.

CORRESPONDENCE Gabriel Zada, Department of Neurological Surgery, Keck School of Medicine, University of Southern California, 1200 North State Street, Ste. 3300 , Los Angeles, CA 90033. email: gzada@usc.edu. 